'Departamento de Neurología, Facultad de Medicina. Pontificia Universidad Católica de Chile. Santiago, Chile.

Sin apoyo financiero.

Recibido el 29 de noviembre de 2019, aceptado el 8 de junio de 2020.

Correspondencia a: Dr. Jorge Tapia Departamento de Neurología Diagonal Paraguay $362,5^{\circ}$ piso. Santiago, Chile. jftapia@med.puc.cl

\section{Hemorragia encefálica por angiopatía amiloidea}

\author{
JORGE TAPIA $^{1}$
}

\section{Amyloid angiopathy brain hemorrhage}

Amyloid angiopathy $(A A)$ is a selective deposition of amyloid in the walls of the brain vessels. It is a form of sporadic and localized amyloidosis, constituted by the A $\beta 4$ protein, the same of Alzheimer's disease senile plaques. The most consistent clinical effect of $A A$ is spontaneous brain hemorrhage $(B H)$. It is the second most common cause of BH after arterial hypertension (HT). Other clinical manifestations are cognitive impairment and transient focal neurological episodes. AA BH is characteristically localized in the cerebral cortex and subcortical white matter (lobar hemorrhage), consistent with the preferential deposit of amyloid in the walls of leptomeningeal and intracortical small cerebral vessels. Other types of $A A$ hemorrhagic complications are microbleeds $(M B)$, cerebral convexity subarachnoid hemorrhage (cSAH) and superficial hemosiderosis (cSS). The diagnosis of $A A B H$ is based on the Boston criteria. Using these criteria, several non-hemorrhagic biomarkers of $A A$ have been identified that can be useful in its diagnosis. The principal AA BH risk factor is age, followed by cSS, $M B$, Apolipoprotein E gen $\varepsilon 2$ and $\varepsilon 4$ alleles, $H T$ and the use of antithrombotics. This condition has a high recurrence rate that shares the same risk factors. There is no specific treatment for $A A B H$. It has a better prognosis than $H T B H$ during the acute period, but worse on the long term, due to its high recurrence rate and cognitive impairment.

(Rev Med Chile 2021; 149: 76-87)

Key words: Cerebral Amyloid Angiopathy; Cerebral Hemorrhage; Stroke; Cerebral Small Vessel Diseases.
$\mathrm{E}$ 1 accidente vascular encefálico (AVE) ha pasado a ser la primera causa de muerte en Chile ${ }^{1}$. En series internacionales, aproximadamente, $80 \%$ de los AVE son isquémicos y $20 \%$ hemorrágicos, $15 \%$ hemorragias intraparenquimatosas o encefálica (HE), $5 \%$ hemorragias subaracnoídeas (HSA) ${ }^{2}$. En Chile, el porcentaje de $\mathrm{HE}$ es mayor, 28 a $35 \%$ en series hospitalarias ${ }^{3} \mathrm{y}$ $25,6 \%$ en un estudio poblacional ${ }^{4}$.

En las últimas décadas ha ocurrido un importante avance en el tratamiento de los AVE isquémicos y HSA, pero no en el de la HE, cuya mortalidad es aproximadamente $40 \%$ mayor que el AVE isquémico ${ }^{5}$ es el único subtipo de AVE no incluido en las patologías con garantía explícita de salud (GES).
Sobre $80 \%$ de las HE son primarias y su causa más frecuente es la hipertensión arterial (HTA), seguida de la angiopatía amiloidea $(\mathrm{AA})^{6}$. En los últimos años ha ocurrido un gran avance en el conocimiento de la HE por AA, lo que motiva la presente revisión.

\section{Amiloidosis encefálica}

Es una forma localizada de amiloidosis que afecta exclusivamente al encéfalo, siendo excepcional su compromiso en casos de amiloidosis generalizada ${ }^{7}$. La gran mayoría es esporádica, siendo muy infrecuentes las formas hereditarias. El amiliode se deposita en el encéfalo de dos for- 
mas: en el parénquima, como placas seniles y en la pared de los vasos, lo que constituye la angiopatía amiloidea $^{7}$ (Figura 1). La proteína que compone el amiloide encefálico es mayoritariamente la $\mathrm{A} \beta_{4}$. En esta revisión se utilizará el término angiopatía amiloidea como equivalente a AA esporádica por $\mathrm{A} \beta_{4}$.

En numerosas entidades clínicas se puede encontrar una $\mathrm{AA}$, siendo la más destacada la $\mathrm{HE}$ primaria por $\mathrm{AA}^{7}$. El $85 \%$ de los casos de enfermedad de Alzheimer (EA) tiene AA, pero solo en el $25 \%$ es grave ${ }^{8}$. La AA fue descrita por W. Scholz en $1938^{9}$, pero solo en la década $1980-89$ se la reconoce como causa de HE. La Tabla 1 muestra la escala de gravedad del depósito amiloide en la $\mathrm{AA}^{10}$. En un estudio de 153 encéfalos con AA, solo aquellos con HE tenían una AA grado IV, y todos ellos un grado III, mientras que en los sin $\mathrm{HE}$, ninguno tenía un grado IV y solo $8 \%$ tenían un grado III $^{10}$. Así, en otro estudio, la especificidad diagnóstica de la biopsia cortical cerebral para establecer que una $\mathrm{HE}$ es por AA es de 99 a $100 \%$ para el grado IV, y 93 a $96 \%$ para el grado III ${ }^{11}$.

La proteína $A \beta_{4}$ es producida en el sistema nervioso central (SNC), principalmente por las neuronas. La Figura 2 muestra su producción, eliminación y depósito en el SNC. El depósito de amiloide en la AA es segmentario en las arterias de mediano y pequeño calibre, de preferencia leptomeníngeas y corteza cerebral, rara vez del cerebelo y excepcionalmente en los núcleos grises centrales y troncoencéfalo, sin comprometer los vasos de la sustancia blanca ${ }^{11}$. Por esto, cuando se opera una HE lobular cerebral, se deben tomar muestras de leptomeninges y corteza cerebral, y hacer cortes seriados del tejido.

La AA puede producir un espectro de lesiones encefálicas (Tabla 2, Figura 3) 3,12 $^{8}$.

\section{Hemorragia encefálica por AA}

Es la complicación más frecuente y conocida de la $\mathrm{AA}^{12}$. De las HE espontáneas, 56 a $81 \%$ son por $\mathrm{HTA}^{13}$, y en el resto, la causa más frecuente es la AA, llegando a $34 \%$ en series de adultos mayores $^{7,13,14}$. Su localización preferente son los lóbulos de los hemisferios cerebrales (HE cerebral lobular) (Figura 4). En una serie anatomopatológica de 171 hematomas por AA, 95,8\% era lobular cerebral, $3,5 \%$ en los núcleos grises centrales, y $1,2 \%$ en el

Tabla 1. Escala de Vonsattel de gravedad de angiopatía amiloidea ${ }^{14}$

\begin{tabular}{|ll|}
\hline Grado I o leve & Depósito de amiloide en la capa media sin alteración de la estructura de la pared vascular \\
\hline Grado II o moderada & Reemplazo de la capa media por amiloide \\
\hline Grado III o grave & Reemplazo de la capa media por amiloide y fragmentación de la pared \\
\hline Grado IV & Necrosis fibrinoide de la pared con trazas de amiloide \\
\hline
\end{tabular}

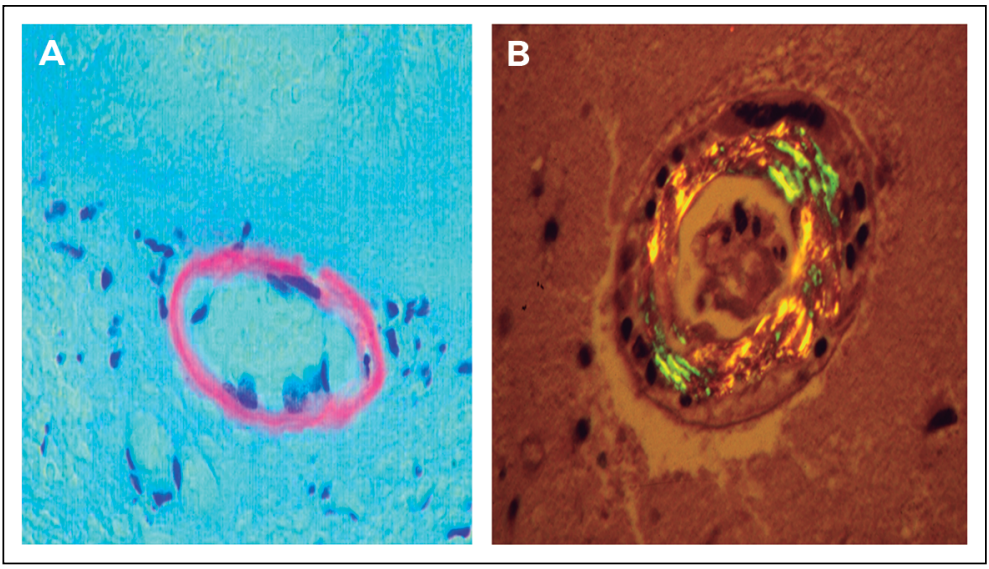

Figura 1. Arteria pequeña de la corteza cerebral con angiopatía amiloidea. A: tinción de rojo del Congo tiñe el amiloide de color pardo rojizo (por lo que también se denomina angiopatía congofílica). B: tinción rojo del Congo bajo la luz polarizada muestra el característico dicroísmo verde-amarillo del amiloide (colección personal del autor). 
cerebelo ${ }^{15}$. Si bien su porcentaje es mayor a nivel frontal, en proporción a la superficie cortical cerebral su frecuencia es mayor a nivel occipital, consistente con el mayor depósito de amiloide en los vasos de este lóbulo, y luego, en orden decreciente, temporal, frontal y parietal ${ }^{10,12,16}$. El $85 \%$ de las hemorragias cerebelosas espontáneas son hipertensivas, y de preferencia de ubicación profunda en los hemisferios cerebelosos (núcleos dentados), mientras de las superficiales, córtico-subcorticales hemisféricas y vermis cerebeloso, un tercio se ha relacionado con $\mathrm{AA}^{17}$. En estudios de tomografía

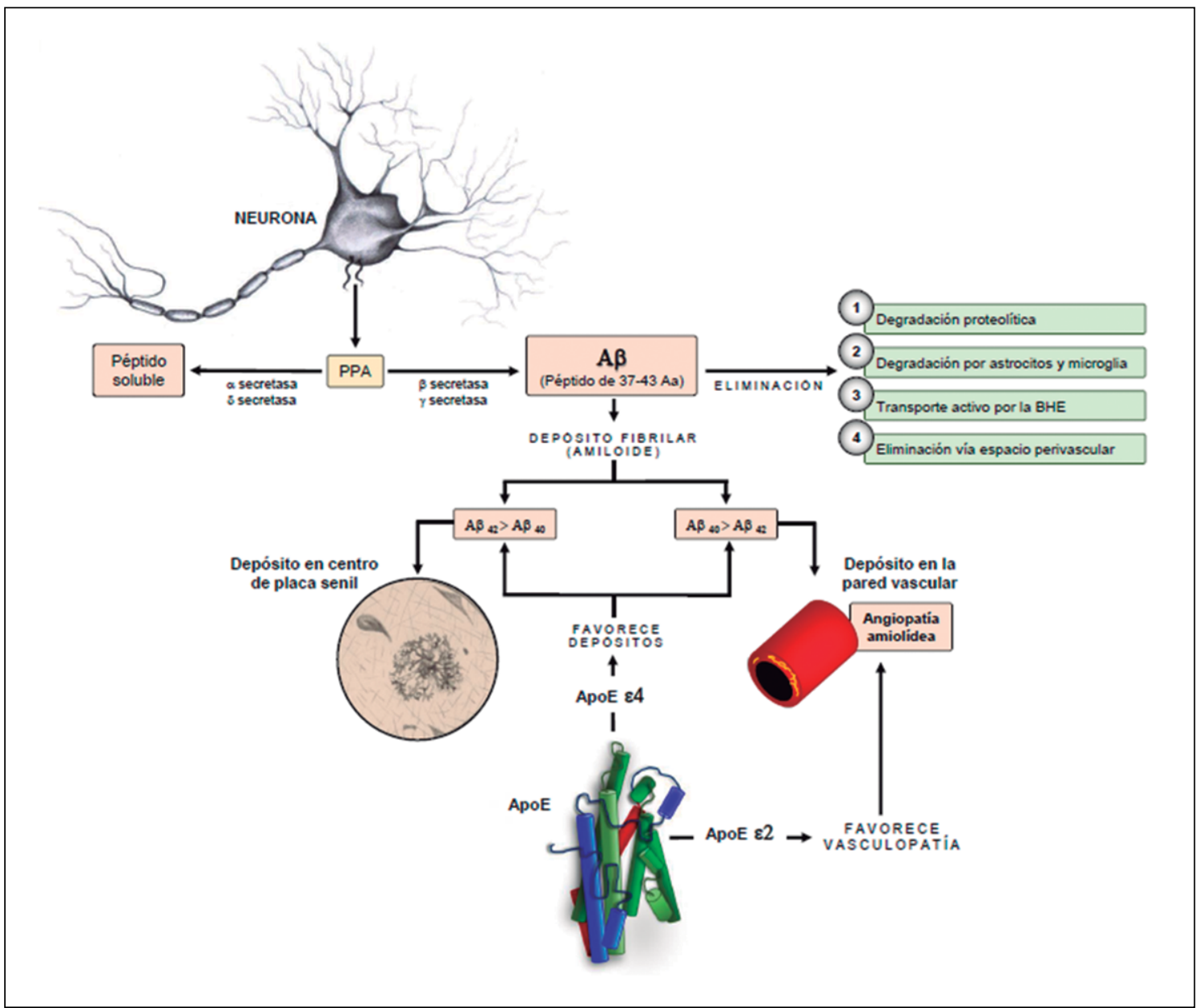

Figura 2. Producción, eliminación y depósito del amiloide $A \beta_{4}$ en el sistema nervioso central ${ }^{8}$. La PPA es una proteína transmembrana que es proteolizada por dos vías: mayoritariamente por clivaje secuencial por la $\alpha$ secretasa y luego la $\gamma$ secretasa, generando un péptido soluble, y en una mucho menor proporción por clivaje secuencial por la $\beta$ secretasa y luego la $\gamma$ secretasa, que la corta a diferentes niveles generando péptidos de 37 a $43 \mathrm{Aa}\left(\mathrm{A} \beta_{4}\right)$. De ellos, los más frecuentes son los de $40\left(A \beta_{40}\right)$ y 42 $\left(A \beta_{42}\right)$ aminoácidos. El $A \beta_{4}$ tiene cuatro vías principales de eliminación: degradación proteolítica, degradación por astrocitos y microglia, eliminación a través de la BHE, y eliminación a través de los espacios perivasculares (Virchow-Robin). Con la edad, aparentemente no por mayor producción, sino por falla en los procesos de eliminación, el $A \beta_{4}$ se acumula y forma fibrillas insolubles que se depositan como amiloide en el centro de la placa senil y en la pared de los vasos (AA). El A $\beta_{42}$ se deposita principalmente en la placa senil y el $A \beta_{40}$ en la pared de los vasos. La ApoE participa en todo este proceso, y su gen tiene los alelos $\varepsilon 2, \varepsilon 3$ y $\varepsilon 4$ con efecto dosis dependiente en el riesgo de desarrollo y severidad de la AA. El alelo $\varepsilon 4$ favorece el depósito de amiloide en los vasos (y también en la placa senil), y el $\varepsilon 2$ favorece los cambios vasculopáticos de la AA (grados III y IV), relacionados a la rotura vascular y hemorragia encefálica. PPA: proteína precursora del amiloide; Aa: aminoácido; BHE: barrera hematoencefálica; AA: angiopatía amiloidea; ApoE: apolipoproteina E (Figura cortesía Dra. B. Wipe). 
Tabla 2. Espectro de lesiones encefálicas de la angiopatía amiloidea ${ }^{8,12}$

Hemorragias
Macrohemorragia
Microhemorragia cerebral cortical
Hemorragia subaracnoidea de la convexidad cerebral
Hemosiderosis superficial de la convexidad cerebral
Microinfarto cerebral cortical
Alteraciones de la sustancia blanca hemisférica cerebral
Alteraciones inflamatorias
Infiltrado inflamatorio perivascular
Vasculitis

computada de encéfalo (TC), las HE cerebrales lobulares por AA, por rotura de vasos intracorticales cerebrales y leptomeníngeos, aproximadamente, $90 \%$ se abre al espacio subaracnoídeo, mientras que las hipertensivas en esta ubicación, por rotura de vasos de la sustancia blanca subcortical, solo en el $40 \%$ se abre al espacio subaracnoideo, elemento que es de utilidad para el diagnóstico de la causa de una hemorragia en esta localización (Figura $4)^{18}$. Las HE cerebrales lobulares por AA pueden ser simultáneamente múltiples. Descartado que sean traumáticas o secundarias a trastornos de la coagulación, metástasis, endocarditis infecciosa, trombosis venosa, se debe pensar como causa una AA, especialmente en pacientes mayores de 60 años, ya que las por causa hipertensiva solo muy excepcionalmente son simultáneamente múltiples ${ }^{19}$.

\section{Microhemorragia encefálica por angiopatía amiloidea}

Corresponden a pequeñas hemorragias intraparenquimatosas que se identifican como focos de hipointensidad de 2 a $10 \mathrm{~mm}$ en la secuencia T2 gradiente de la RM $\left(\mathrm{RM} \mathrm{T}^{*}\right)^{20}$. La secuencia de susceptibilidad magnética por RM (RM SWI), con cortes finos, permite ver tres veces más microhemorragias $(\mathrm{MH})$, y es la secuencia recomendada por la American Stroke Association (ASA) para

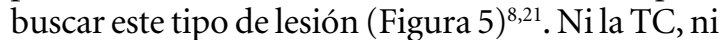
la $\mathrm{RM}$ sin estas secuencias muestran las $\mathrm{MH}^{8,21}$. Su prevalencia en estudios poblacionales con $\mathrm{RM} \mathrm{T}^{*}$ es de 3,1 a 23,5\%, correspondiendo los porcentajes más altos a estudios de pacientes de mayor edad y

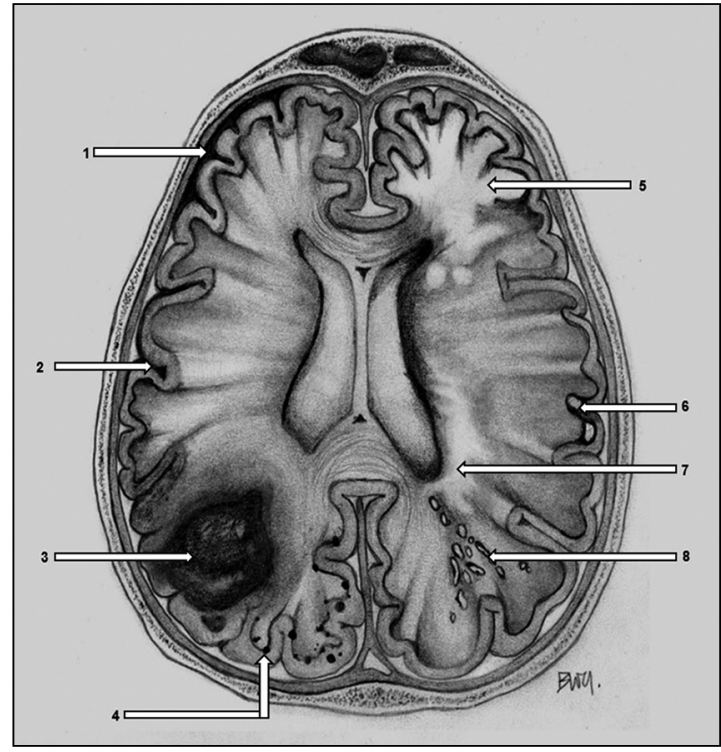

Figura 3. Corte axial hemisferios cerebrales a nivel del centro oval que muestra el espectro de lesiones por angiopatía amiloidea. En el hemisferio a izquierda se muestran las lesiones hemorrágicas: 1: hemorragia subaracnoidea de la convexidad cerebral; 2: hemosiderosis superficial de la convexidad cerebral; 3: macrohemorragia (hematoma lobular); 4: microhemorragias cerebrales corticales. En el hemisferio a derecha alteraciones no hemorrágicas; 5: vasculitis (alteración sustancia blanca); 6: microinfarto cerebral cortical; 7: focos de alteración de la sustancia blanca hemisférica cerebral; 8: dilatación de los espacios de Virchow-Robin (ilustración cortesía Dra. Bárbara Wipe).

RM con cortes más finos y técnicas ecoplanares o de tres dimensiones ${ }^{20}$. Las $\mathrm{MH}$ se clasifican según su localización en superficiales, córtico-subcortical en la corteza cerebral y profundas, sustancia blanca profunda de los hemisferios cerebrales, núcleos grises centrales, troncoencéfalo y cerebelo (Figura $5)^{20}$. Las estrictamente superficiales, que pueden incluir las cerebelosas, se relacionan con AA, con un valor predictivo positivo en la patología de $87,5 \%{ }^{22}$, mientras las profundas, y mixtas (superficiales y profundas), se relacionan con HTA, sin poder descartar la AA en estas últimas, especialmente cuando la proporción de superficiales es muy superior a las profundas ${ }^{23}$. La mayoría de las $\mathrm{MH}$ por AA son asintomáticas, pudiendo manifestarse clínicamente como deterioro cognitivo y episodios de alteración neurológica focal transitorios $(\mathrm{ENFT})^{20,24}$. 

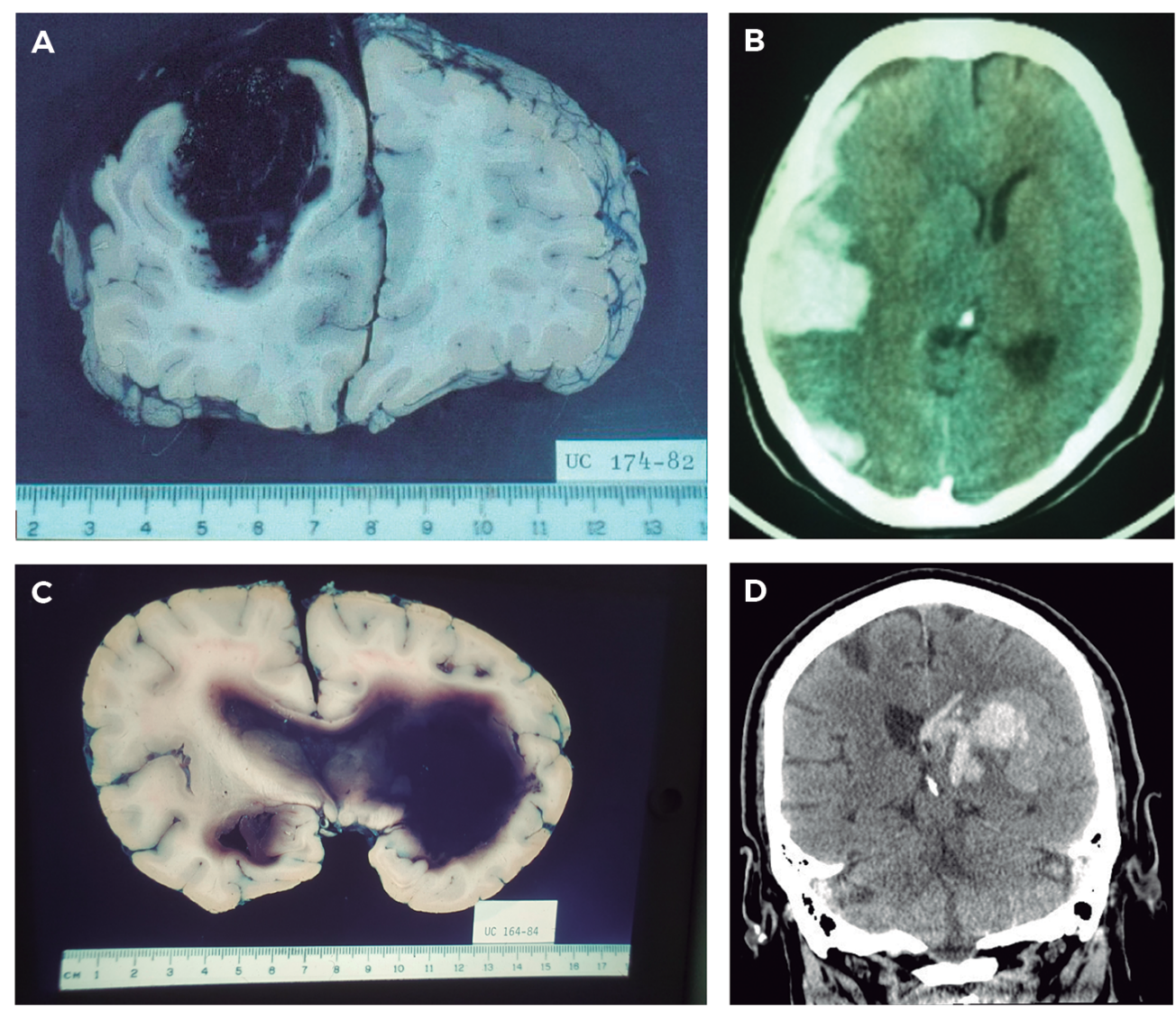

Figura 4. A: corte coronal lóbulos frontales por delante del cuerpo calloso que muestra una hemorragia córtico-subcortical amiloidea (hemorragia encefálica lobar o lobular), que se abre al espacio subaracnoideo suprayacente. B: corte axial de tomografía computarizada de cerebro que muestra una hemorragia amiloidea frontal lobular abierta al espacio subaracnoideo suprayacente. C: Corte coronal lóbulos frontales que muestran una hemorragia hipertensiva putaminal que se extiende hacia arriba y lateral, a la sustancia blanca lobular, que se abre hacia el ventrículo lateral respetando la corteza cerebral, sin abrirse al espacio subaracnoideo. D: Corte coronal de tomografía computarizada de cerebro que muestra una hemorragia hipertensiva putaminal con similares características respecto a la fotografía C.

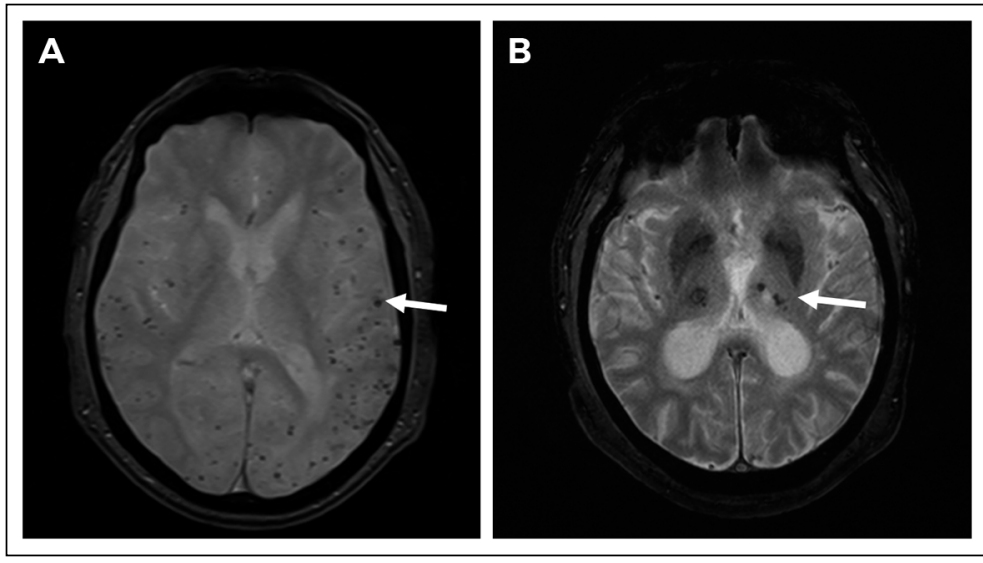

Figura 5. Cortes axiales de resonancia magnética en secuencia de suceptibilidad magnética (RM SWI). A: muestra múltiples pequeños focos negros exclusivamente en la corteza cerebral correspondientes a microhemorragias $(\mathrm{MH})$ (flecha) por angiopatía amiloidea. B: MH exclusivamente en núcleos grises centrales (tálamos y lenticulares) (flecha) hipertensivas (cortesía Dr. Juan Pablo Cruz). 


\section{Hemorragia subaracnoidea espontánea de la convexidad cerebral por angiopatía amiloidea}

Corresponde a una HSA de la convexidad de la corteza cerebral (HSAc), sin extensión a la fisura interhemisférica, cisternas silvianas o de la base, ni a los ventrículos, como ocurre en la HSA aneurismática (Figura 6A) ${ }^{25}$. Se considera secundaria a la rotura de una pequeña arteria leptomeníngea por AA. En una serie de 389 casos de HSA espontánea, 29 (7,45\%) correspondieron a HSAc. En 10 de ellos, todos mayores de 60 años, el diagnóstico final fue $\mathrm{AA}^{25}$. Su manifestación clínica más frecuente fue como ENFT, siendo infrecuente la cefalea y su recurrencia clínica ${ }^{25,26}$. En la Tabla 3 se muestran los diagnósticos diferenciales de la HSAc por $\mathrm{AA}^{25,26}$.

\section{Tabla 3. Diagnóstico diferencial de la hemorragia subaracnoidea espontánea de la convexidad cerebral $^{25,26}$}

- Angiopatía amiloidea
- Síndrome de vasoconstricción arterial segmentaria
reversible
- Trombosis de vena cortical
- Síndrome de hiperperfusión encefálica
- Coagulopatía
- Consumo de drogas de uso ilícito (cocaína, anfetaminas)
- Vasculitis
- Endocarditis infecciosa
- Fístula dural

\section{Hemosiderosis superficial cortical cerebral por angiopatía amiloidea}

Corresponde al depósito de hemosiderina subpial y en las capas superficiales subyacentes de la corteza de la convexidad cerebral (HSSc) (Figura $6 \mathrm{~B})^{27}$. Se considera que es secundaria a una HSAc ${ }^{28}$. Está presente en el 40 a $60 \%$ de las HE por AA, en 0 a $4 \%$ de las HE por otras causas, y en el $0,7 \%$ de la población general ${ }^{28,29}$. Su manifestación clínica más frecuente, aproximadamente en el $50 \%$ los casos, es como ENFT ${ }^{12,28-30}$.

Las presentaciones clínicas más frecuentes de las complicaciones hemorrágicas de la AA son ${ }^{12}$ :

1. AVE hemorrágico como HE lobular. Forma de presentación clínica más frecuente ${ }^{14,31}$. Según su tamaño y localización se manifestará por síntomas y signos generales de la $\mathrm{HE}$, y focales según el lóbulo comprometido.

2. Deterioro cognitivo. Forma de presentación clínica que puede preceder en 25 a $40 \%$ a la HE lobular ${ }^{15}$. Se considera secundario a las múltiples alteraciones encefálicas referidas en la Tabla 2, y cuyo perfil cognitivo es más parecido al deterioro cognitivo vascular que al de la $\mathrm{EA}^{12}$.

3. Episodios de alteración neurológica focal transitorios. Forma de presentación en aproximadamente el 15\% de los casos (Tabla 4) ${ }^{24,30}$. Son semejantes a las auras jaquecosas, y como estas, su patogenia más probable son ondas de depolarización cortical propagada. Puede confundirse con crisis isquémicas transitorias e indicarse antitrombóticos, que no son de utilidad, y pueden ser de riesgo en la AA. Por

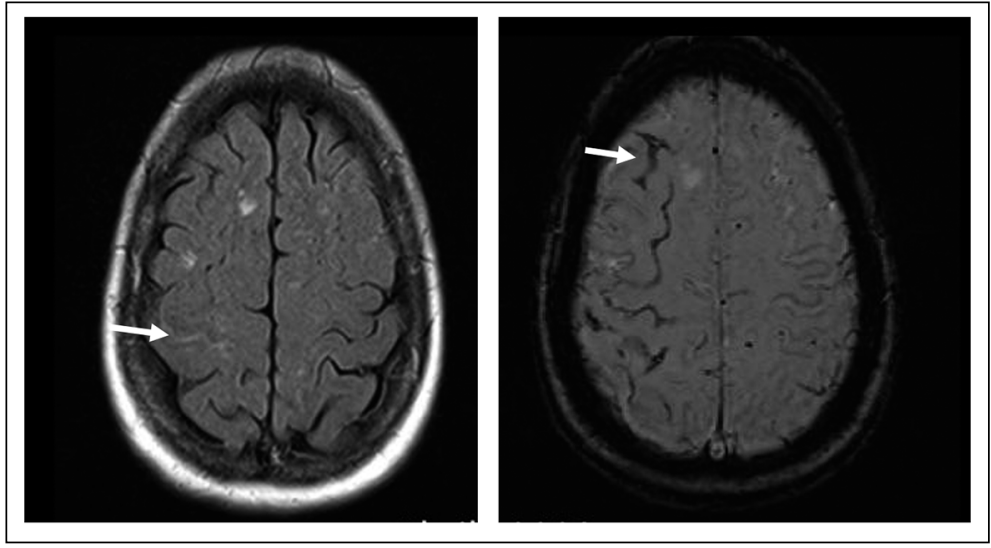

Figura 6. Cortes axiales de resonancia magnética a nivel del centro oval. A: Secuencia T1 muestra hemorragia subaracnoidea de color blanco ocupando los surcos de la convexidad cerebral (flecha). B: Secuencia suceptibilidad magnética muestra en color negro hemosiderosis superficial en los surcos de la convexidad cerebral (flecha) (cortesía Dr. Juan Pablo Cruz). 
Tabla 4. Criterios diagnósticos de episodios neurológicos focales transitorios por angiopatía amiloídea 24,30

- Al menos uno de los siguientes síntomas neurológicos focales transitorios de minutos de duración (típicamente menos de $30 \mathrm{~min}$ ), recurrentes, estereotipados: sensitivos como parestesias con marcha que va extendiéndose gradualmente dentro de una extremidad como a otra extremidad y cara, motores, principalmente paresia y con menos frecuencia movimientos como aleteo de una extremidad, visuales como fosfenos o deficitarios como escotomas

- RM T2* o SWI muestra dos o más microhemorragias estrictamente superficiales, macrohemorragia cerebral lobular única o múltiple, aceptándose que ambos tipos de hemorragia pueden ser cerebelosas, hemorragia subaracnoídea de la convexidad cerebral, siderosis superficial de la convexidad cerebral

- Ausencia de causas alternativas de estas hemorragias

- Exclusión de otras causas de episodios neurológicos focales transitorios

- Edad mayor a 55 años

esto, el estudio de las alteraciones neurológicas focales transitorias debe incluir una RM con secuencias $\mathrm{T} 2{ }^{\star}$ o SWI.

\section{Criterio diagnóstico de hemorragia por angiopatía amiloidea}

La Tabla 5 muestra los criterios de Boston modificados ${ }^{19}$. Cabe destacar que la HTA no es un criterio de exclusión; la presencia de hemorragia cerebelosa no cuenta como lesión hemorrágica, pero no invalida el diagnóstico; el deterioro cognitivo no está incluido, ya que precede, aproximadamente, a $25 \%$ de los casos y tampoco la determinación de los alelos $\varepsilon 2$ y $\varepsilon 4$, ya que solo están presentes en un subgrupo de pacientes ${ }^{8}$. Este criterio ha sido validado en estudios hospitalarios, teniendo una sensibilidad de 71,1 a $76,9 \%$ y una especificidad de 87,5 a $100 \%$, y $100 \%$ de sensibilidad en casos de AA hereditaria holandesa ${ }^{23}$.

\section{Factores de riesgo de $H E$ por $A A$}

Los factores de riesgo más frecuentes son:

Edad: Principal factor de riesgo de AVE, particularmente de HE por $\mathrm{AA}^{5}$. En series de autopsia, la AA aumenta de aproximadamente $10 \%$ entre los 60 y 69 años, a 50\% en mayores de 90 años ${ }^{7,11,12}$. La Tabla 6 muestra cómo la localización y la edad del paciente orientan a la posible causa de una HE. Las cuatro localizaciones en relación a HTA corresponden a los llamados sitios típicos de HE, mientras que las hemorragias cerebrales lobulares son llamadas en sitio atípico, y requieren otras
Tabla 5. Criterios de Boston para el diagnóstico de angiopatía amiloidea*

AA definitiva

Estudio de autopsia que muestre:

- Hemorragia cerebral lobular ${ }^{a}$ cortical o córtico-subcortical

- AA grave

- Ausencia de otra lesión diagnóstica

AA probable con patología

Clínica y biopsia que muestre:

- Hemorragia cerebral lobulara, cortical o córtico-subcortical

- Algún grado de AA en la biopsia

- Ausencia de otra lesión diagnóstica

AA probable

Clínica y RM o TC que muestre

- Hemorragias cerebrales lobularesa, corticales o córticosubcorticales, múltiples (se permite hemorragia cerebelosa), o única más HSSc o HSAc

- Edad mayor de 55 años

- Ausencia de otras causas de hemorragia ${ }^{b}$

AA posible

Clínica y RM o TC que muestre

- Hemorragia cerebral lobulara, cortical o córtico subcortical, única, o HSSc o HSAc

- Edad mayor de 55 años

- Ausencia de otras causas de hemorragiab

AA: angiopatía amiloidea; RM: resonancia magnética de cerebro; TC: tomografía computarizada de cerebro; HSSc: hemosiderosis superficial de la convexidad cerebral; HSAc: hemorragia subaracnoidea de la convexidad cerebral. ahemorragia cerebral lobular incluye macro y microhemorragia; 'diagnóstico diferencial de hemorragia cerebral lobular: antecedente de traumatismo encéfalo-craneano, infarto con transformación hemorrágica; malformación vascular; tumor con hemorragia; terapia con warfarina e INR mayor de 3; vasculitis; trombosis venosa. *Modificada de Charidimou A, Gang Q, Werring D. J Neurol Neurosurg Psychiatry 2012; 83: $124-37^{12}$. 
Tabla 6. Relación entre localización de la hemorragia encefálica, edad del paciente y causas más probables

\begin{tabular}{|llc|}
\hline Causa & Localización más frecuente & Edad más frecuente (años) \\
\hline Hipertensión arterial crónica & Putamen, tálamo, cerebelo, protuberancia & $45-65$ \\
\hline Drogas de uso ilícito & Lóbulos cerebrales & $<45$ \\
\hline Malformaciones vasculares & Lóbulos cerebrales & $>45$ \\
\hline Angiopatía amiloídea & Lóbulos cerebrales & $>70$ \\
\hline Tumor & Lóbulos cerebrales & $>50$ \\
\hline Alteración coagulación & Lóbulos cerebrales, cerebelo & Cualquier edad \\
\hline
\end{tabular}

consideraciones causales. De las HE espontáneas hipertensivas, solo 5 a $15 \%$ son cerebrales lobulares $^{6}$. En series patológicas, 26 a $62 \%$ de las HE cerebrales lobulares son hipertensivas ${ }^{32}$, pero en mayores de 70 años, 50 a $75 \%$ son por $\mathrm{AA}^{31,32}$. El caso más joven publicado de HE que con certeza correspondía a una AA esporádica tenía 53 años ${ }^{8}$. El criterio de Boston establece que el paciente tenga 55 o más años ${ }^{19}$.

Factores genéticos: El polimorfismo del gen de la ApoE4 es el único factor genético identificado en la $\mathrm{AA}^{8}$. Los alelos $\varepsilon 2$ y $\varepsilon 4$ tienen una frecuencia significativamente mayor en la hemorragia definitiva o probable de acuerdo al criterio de Boston ${ }^{33}$.

Hemosiderosis superficial de la convexidad cerebral: Es el factor de riesgo más importante, aumentando en cuatro veces el riesgo de una primera $\mathrm{HE}$ cerebral lobular por $\mathrm{AA}^{28}$.

Microhemorragia: Las estrictamente superficiales son un importante factor de riesgo de una primera HE cerebral lobular por AA, con $5 \%$ anual respecto a $0,015-0,05 \%$ en la población general de personas mayores ${ }^{34}$.

Hipertensión arterial: Si bien tiene una alta prevalencia poblacional, que aumenta con la edad, se considera que es un factor de riesgo independiente de HE cerebral lobular por AA. La HTA favorece el depósito de amiloide cerebral ${ }^{35}$. En pacientes con AA confirmada por patología, 50 a 59\% de aquellos con HE cerebral lobular eran hipertensos, mientras $23 \%$ lo eran en cuadros sin hemorragia $^{10,32}$. La reducción de la presión arterial en pacientes con historia de AVE disminuye en
$77 \%$ el riesgo de una HE por $\mathrm{AA}^{36}$. No se ha descrito relación de otros factores de riesgo vascular con AA.

Antitrombóticos: El ácido acetilsalicílico (AAS), en estudios poblacionales, aumenta en 2,7 veces la prevalencia de $\mathrm{MH}$ estrictamente superficiales $^{37}$. También aumenta significativamente el riesgo de $\mathrm{HE}$ en pacientes con $\mathrm{MH}$, especialmente si son estrictamente superficiales ${ }^{38}$. Los antiplaquetarios son un factor de riesgo independiente de mayor volumen de HE cerebral lobular primaria $^{39}$. La warfarina aumenta el riesgo de HE en la AA. Esta afirmación se basa en las siguientes características de las $\mathrm{HE}$ en relación al uso de warfarina: en su mayoría son lobulares $(69 \%$ versus $31 \%$ profundas), sobre dos tercios ocurren con niveles terapéuticos de INR, en el 64\% de los pacientes mayores de 65 años en los que se hace estudio patológico se encuentra una $\mathrm{AA}$, las $\mathrm{MH}$ estrictamente superficiales y el alelo $\varepsilon 2$ aumenta en 3,8 veces el riesgo de esta complicación ${ }^{40-42}$. No obstante lo anterior, un modelo de análisis de Markov considera que las $\mathrm{MH}$, o los alelos $\varepsilon 2$ y $\varepsilon 4$ de la ApoE, si bien confieren mayor riesgo de $\mathrm{HE}$ en relación al uso de anticoagulantes orales, no los contraindican ${ }^{43}$. Las guías de la ASA recomiendan el uso de anticoagulantes orales cuando están indicados, independiente de la presencia de $\mathrm{MH}, \mathrm{y}$ mas aún, no consideran que se deba practicar una RM previo a su indicación ${ }^{21}$.

Tromboliticos: Las MH aumentan en 2,36 veces el riesgo de $\mathrm{HE}$ posterior a trombólisis intravenosa del AVE isquémico ${ }^{44}$. Las $\mathrm{MH}$ están presentes en 12 a 39\% de los pacientes con AVE isquémico elegibles para trombólisis ${ }^{45}$, y el $70 \%$ 
de los pacientes con HE postrombólisis de un AVE isquémico, presentan en la patología una $\mathrm{AA}^{46}$. No obstante lo anterior, y considerando que la TC no muestra las $\mathrm{MH}$, las guías de la ASA no recomiendan practicar una RM previo al uso de trombolíticos intravenosos en el AVE isquémico ${ }^{47}$.

\section{Recurrencia de la hemorragia encefálica por angiopatía amiliodea}

Su recurrencia anual es de aproximadamente $10 \%{ }^{8,12,48-50}$.

Los principales factores de riesgo son:

Hemosiderosis superficial de la convexidad cerebral: Después del antecedente de haber presentado una $\mathrm{HE}$ por $\mathrm{AA}^{49}$, es el principal factor de riesgo de recurrencia ${ }^{51}$.

Microhemorragia superficial: El riesgo aumenta progresivamente en relación a su número ${ }^{52}$.

Factores genéticos: Los alelos $\varepsilon 2$ y $\varepsilon 4$ de la ApoE, aumentan en aproximadamente cuatro veces el riesgo de recurrencia ${ }^{50}$.

Antitrombóticos: El uso de antiplaquetarios luego de una HE cerebral lobular por AA aumenta en, aproximadamente, cuatro veces su recurren$\operatorname{cia}^{49}$. La recomendación de las guías de la ASA es considerar la indicación de antiplaquetarios en monoterapia cuando su uso es indispensable $e^{53}$. Los anticoagulantes orales, específicamente la warfarina, no han sido bien estudiados, dado que las series excluyen a los pacientes con $\mathrm{HE}$ previa. Asimismo, las escalas de riego de AVE en la fibrilación auricular no valvular (FANV), no incluyen el parámetro de antecedente de HE. Un análisis en base al modelo de decisión de Markov, no recomienda utilizar anticoagulantes luego de una HE cerebral lobular por AA, dado el alto riesgo de recurrencia ${ }^{54}$, y así lo aconsejan las guías de la ASA $^{53}$. Sin embargo, un estudio observacional de pacientes con FANV, a los que se les reanudó el anticoagulante luego de una HE posible o probable por AA, según el criterio Boston, al año de seguimiento tuvieron una significativa menor mortalidad y morbilidad ${ }^{55}$,

Hipertensión arterial: El mal control de la HTA aumenta la recurrencia en 3,53 veces a tres años ${ }^{56}$,

Cirugía: Publicaciones de 1980-89 recomendaban no operar cuando se sospechaba una HE por AA por el riesgo de sangrados durante y en el postoperatorio. En series quirúrgicas posteriores, este riesgo no fue significativo, por lo que actual- mente se considera que la cirugía puede realizarse cuando está indicada ${ }^{57}$.

\section{Biomarcadores de angiopatía amiloídea}

Corresponden a diversas alteraciones no hemorrágicas detectadas en exámenes de laboratorio, asociadas AA, que eventualmente podrían incluirse en el criterio diagnóstico, mejorando su sensibilidad, sin disminuir la especificidad (Tabla 7$)^{23}$.

En la RM, las hiperintensidades de la sustancia blanca, como la dilatación de los espacios de Virchow-Robin, son de localización cerebral subcortical (centro semioval), a diferencia de aquellas por microangiopatía hipertensiva, de localización profunda en los hemisferios cerebrales y protuberancia ${ }^{8,12,58}$. Los microinfartos cerebrales corticales pueden no verse en la RM, reportándose en $23 \%$ de los casos en el período agudo de una HE por AA versus el $8 \%$ de las HE hipertensivas ${ }^{8,59}$. La disminución del grosor de la corteza cerebral se ve en pacientes con AA y sin demencia, comparado con controles sanos ${ }^{12}$. La RM por difusión en tensor permite ver los tractos de fibras en los hemisferios cerebrales. Los pacientes con AA definitiva o probable presentan, con esta técnica, una alteración de los tractos que proyectan a las cortezas occipitales, parietales $\mathrm{y}$ temporales posteriores, $\mathrm{y}$ esto se relaciona a mayor carga de amiloide en la tomografía por emisión de positrones (PET), que clínicamente se caracteriza por peor desempeño en pruebas de velocidad de pensamiento, funciones ejecutivas y marcha, como se ve en las demencias vasculares subcorticales ${ }^{12,23}$. En estudios con RM funcional

Tabla 7. Principales biomarcadores de angiopatía amiloidea $^{23}$

RM:

- Hiperintensidades de la sustancia blanca

- Dilatación de los espacios de Virchow-Robin

- Microinfartos cerebrales corticales

- Disminución del grosor de la corteza cerebral

- Menor conectividad cerebral analizada por difusión por tensor

- Menor reactividad vascular

PET para amiloide

Estudio del LCR

RM: resonancia magnética de cerebro; PET: tomografía por emisión de positrones; LCR: líquido cefalorraquídeo. 
se evidencia una menor respuesta al realizar tareas visuales (corteza occipital), no así con tareas motoras (frontal). Sin embargo, los potenciales evocados visuales son normales, lo que orienta a una alteración del funcionamiento de la unidad neurovascular por menor reactividad vasodilatadora en las regiones posteriores, consistente con el mayor depósito de amiloide en los vasos a este nivel, pero sin disminuir el flujo sanguíneo basal con mantención de la actividad metabólica neuronal evidenciada por los potenciales evocados visuales ${ }^{12,23}$.

En la PET para detectar amiloide se puede usar el ligando Pittsburgh Compound B ( $\mathrm{PiB}$ ) o el florbetapir ${ }^{12,23,60}$. El depósito amiloide en la corteza cerebral no es específico, también ocurre en la enfermedad de Alzheimer, demencia por cuerpos de Lewy, y en personas sanas a mayor edad (10\% en mayores de 65 años, 55\% en los mayores de 82 años). Así la PET con PiB tiene 91\% sensibilidad y $55 \%$ de especificidad en la detección AA respecto a control sano, y el florbetapir $100 \%$ sensibilidad, $89 \%$ especificidad en la detección de una HE cerebral lobular por $\mathrm{AA}^{60}$. Un estudio negativo con estos ligandos descarta la presencia de una AA grado III, lo que hace altamente improbable que una HE sea por una $\mathrm{AA}^{12}$. La PET para amiloide es útil en el diagnóstico en casos de hemorragia por AA posible según el criterio de Boston, especialmente en MH o HSSc sin HE, casos de hemorragias mixtas, superficiales y profundas, y hemorragia cerebelosa en que se sospecha sea por $\mathrm{AA}^{12,60}$.

En el líquido cefalorraquídeo, la $\beta_{40}$ está significativamente disminuida en la $\mathrm{AA}$ en relación a la enfermedad de Alzheimer, y la $\beta_{42}$ significativamente disminuida en la AA versus controles $\operatorname{sanos}^{23,61}$. La proteína tau está aumentada en la AA,

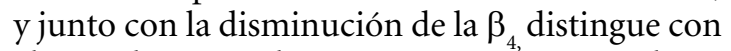
alto grado certeza los pacientes con esta patología de controles sanos ${ }^{61}$.

\section{Pronóstico de la hemorragia encefálica por angiopatía amiloidea}

No existe un tratamiento específico para la AA. En comparación con la HE hipertensiva, la por AA tiene factores favorables, como ser de ubicación superficial, no abrirse al sistema ventricular y tener menor mortalidad (10 a 30\% versus $40 \%)^{5}$. Pero tiene factores desfavorables, como presentarse en pacientes de más edad y tener mayor volumen, recurrencia anual y demencia posterior, la que al año es de $23,4 \%$ versus 9,2\% en la HE hipertensiva, llegando a $40 \%$ a largo plazo $^{49}$.

\section{Referencias}

1. Ministerio de Salud de Chile. Plan de acción Ataque Cerebrovascular. Segunda Edición, 2014. Disponible en: www.redcronicas.cl/wp-content/uploads/2014/03/ Plan-de-accion-AtaqueCerebrovascular-2014.pdf.

2. Feigin VL, Lawes CM, Bennett DA, Anderson CS. Stroke epidemiology: a review of population-based studies of incidence, prevalence, and case-fatality in the late 20th century. Lancet Neurol 2003; 2 (1): 43-53.

3. Tapia J, Corbalán R, Huete I, Valdés F, Villanueva C, Córdoba J, et al. Estudio multidisciplinario del accidente vascular encefálico: I.- definición, diseño y resultados generales. Rev Med Chile 1992; 120: 1414-22.

4. Lavados P, Sacks C, Prina L, Escobar A, Toss C, Araya $\mathrm{F}$, et al. Incidence, 30 days case-fatality rate and prognosis of stroke in Iquique, Chile: results of a two years community based prospective study (PISCIS Proyect). Lancet 2005; 365: 2206-15.

5. van Arch C, Luitse M, Rinkel G, van der Tweel I, Algra A, Klijn C. Incidence, case fatality, and functional outcome of intracerebral haemorrhage on time, according to age, sex, and ethnic origin: a systematic review and meta-analysis. Lancet Neurol 2010; 9 (2): 167-76.

6. Gross B, Friedlander B. Cerebral intraparenchymal hemorrhage: a review. JAMA 2019; 321 (13): 1295-303.

7. Tapia J. Enfermedad cerebrovascular aguda hemorrágica en el adulto mayor. En: Barrientos N, Chaná P, Editores, Neurología del Adulto Mayor. Santiago, Chile: Editorial Ediciones de la Sociedad de Neurología, Psiquiatría y Neurocirugía de Chile; 2000. P. 157-79.

8. Charidimou A, Gang Q, Werring D. Sporadic cerebral amyloid angiopathy revisited: recents insights into pathophysiology and clinical spectrum. J Neurol Neurosurg Psychiatry 2012; 83: 124-37.

9. Scholz W. Studien zur Pathologie der Hirngefässel: die drusige Entartung der Hirnarteiren und Capillaren. Gesamte Neurol Psychiatr 1938; 162: 694-715.

10. Vonsattel J, Myers R, Hedley-Whyte E, Ropper A, Bird E, Richardson E. Cerebral amyloid angiopathy without and with cerebral hemorrhages: a comparative histological study. Ann Neurol 1991; 30: 637-49.

11. Greenberg S, Vonsattel J. Diagnosis of cerebral amyloid angiopathy sensitivity and specificity of cortical biopsy. Stroke 1997; 28: 1418-22. 
12. Charidimou A, Boulouis G, Gurol M, Ayata C, Bacskai B, Frosch M, et al. Emerging concepts in sporadic cerebral amyloid angiopathy. Brain 2017; 140 (7): 1829-50.

13. Kase C, Shoamanesh A, Greenberg S, Caplan L. Intracerebral hemorrhage. En: Grotta J, Albers G, Broderick J, Kasner S, Lo E, Mendelow A, Sacco R, Wong L.Wolf P Editores, Stroke Pathophysiology, Diagnosis, and Management. China: Editorial Elsevier; 2016. p. 466-515e12.

14. Biffi A, Anderson C, Jagiella J, Schmidt H, Kissela B, Hansen B, et al. APOE genotype and extent of bleeding and outcome in lobar intracerebral haemorrhage: a genetic association study. Lancet Neurol 2011; 10: 702-09.

15. Vinters H. Cerebral amyloid angiopathy. A critical review. Stroke 1987; 18: 311-24.

16. Rosand J, Muzikansky A, Kumar A, Wisco J, Smith E, Betensky R, et al. Spatial clustering of hemorrhages in probable cerebral amyloid angiopathy Ann Neurol 2005; 58: 459-62.

17. Pasi M, Marini S, Morotti A, Boulouis G, Xiong L, Charidimou A, et al. Cerebellar hematoma location implications for the underlying microangiopathy. Stroke 2018; 49: 207-10.

18. Rodrigues M, Samarasekera N, Lerpiniere C, Humphreys C, McCarron M, White P, et al. The Edinburgh $\mathrm{CT}$ and genetic diagnostic criteria for lobar intracerebral haemorrhage associated with cerebral amyloid angiopathy: model development and diagnostic test accuracy study. Lancet Neurol 2018; 17 (3): 232-40.

19. Greenberg S, Edgar M. Case records of the Massachusetts General Hospital. N Eng J Med 1996; 325: 189-96.

20. Greenberg S, Vernooj M, Cordonnier C, Viswanathan A, Salman R, Warach S, et al. Cerebral microbleeds: a guide to detection and interpretation. Lancet Neurol 2009; 8: 165-74.

21. Smith E, Saposnik G, Biessels G, Doubal F, Fornage M, Gorelick P, et al. Prevention of stroke in patients with silent cerebrovascular disease AHA/ASA Scientific Statement. Stroke 2017; 48: e44-e71.

22. Martínez S, Romero J, Shoamanesh A, McKee A, van Etten E, Ponters O, et al. Diagnostic value of lobar microbleeds in individuals without intracerebral hemorrhage. Alzheimer's \& Dementia 2015; 11: 1480-88.

23. Greenberg S, Charidimou A. Diagnosis of cerebral amyloid angiopathy evolution of the Boston criteria. Stroke 2018; 49: 491-7.

24. Charidimou A, Baron J, Werring D. Transient focal neurological episodes, cerebral amyloid angiopathy, and intracerebral hemorrhage risk: looking beyond TIAs. Int J Stroke 2013; 8: 105-8.

25. Kumar S, Goddeau R, Selim H, Thomas A, Schlaug G, Alhazzani A, et al. Atraumatic convexal subarachnoid hemorrhage clinical presentation, imaging patterns, and etiologies. Neurology 2010; 74: 893-9.

26. Beitzke M, Gattringer T, Enzinger C, Wagner G, Niederkorn K, Fazekas F. Clinical presentation, etiology, and long-term prognosis in patients with nontraumatc convexal subarachnoid hemorrhage. Stroke 2011; 42: 3055-66.

27. Charidimou A, Linn J, Vernooj M, Opherk C, Akoudad S, Baron J, et al. Cortical superficial siderosis: detection and clinical significance in cerebral amyloid angiopathy and related conditions Brain 2015; 138: 2126-39.

28. Charidimou A, Boulouis G, Xiong L, Jessel M, Roongpiboonsopit D, Ayres A et al. Cortical superficial siderosis and first-ever cerebral hemorrhage in cerebral amyloid angiopathy. Neurology 2017; 88: 1607-14.

29. Shoamanesh A, Martínez S, Oliveira J, Reijmer Y, Falcone $G$, Ayres A, et al. Interrelationship of superficial siderosis and microbleeds in cerebral amyloid angiopathy. Neurology 2014; 83: 1838-43.

30. Charidimou A, Peeters A, Gregoire S, Vandermeeren Y, Laloux P, Jäger H, et al. Spectrum of transient focal neurological episodes in cerebral amyloid angiopathy multicenter magnetic resonance imaging cohort study and meta-analysis. Stroke 2012; 43: 2324-30.

31. Samarasekera N, Smith C, Salman A. The association between cerebral amyloid angiopathy and intracerebral haemorrhage: systematic review and meta-analysis. J Neurol Neurosurg Psychiatry 2012; 83: 275-81.

32. Knudsen K, Rosand J, Karluk D, Greenberg S. Clinical diagnosis of cerebral amyloid angiopathy: validation of the Boston criteria. Neurology 2001;56: 537-9.

33. Greenberg S, Vonsattel J, Segal A, Chiu R, Clatworthy B, Liao A, et al. Association of apolipoprotein E 2 and vasculopathy in cerebral amyloid angiopathy. Neurology 1998; 50: 961-5.

34. van Etten E, Auriel E, Haley K, Ayres A, Vashevich A, Schab K, et al. Incidence of symptomatic hemorrhage in patients with lobar microbleeds Stroke 2014; 45: 2280-5.

35. Gottesman R, Schneider A, Zhou Y, Coresh J, Green F, Gupta N, et al. Association between midlife vascular risk factors and estimated brain amyloid deposition. JAMA 2017; 317 (14): 1443-50.

36. Arima H, Tzourio C, Anderson C, Woodward M, Bousser M, MacMahon S, et al. Effects of perindopril-based lowering of blood pressure on intracerebral hemorrhage related to amyloid angiopathy the PROGRESS trial. Stroke 2010; 41: 394-6.

37. Vernooj M, Haag M, van der Lugt A, Hofman A, Krestin $G$, Stricker B, et al. Use of antitrombotic drugs and the presence of cerebral microbleeds the Rotterdam scan study. Arch Neurol 2009; 66 (6): 714-20. 
38. Gregorie S, Jäger H, Yousry T, Kallis C, Brown M, Werring D. Brain microbleeds as a potential risk factor for antiplatelet-related intracerebral haemorrhage: hospital-based, case-control study. J Neurol Neurosurg Psychiatry 2010; 81: 679-84.

39. Falcone G, Rosand J. Aspirin should be discontinued after lobar intracerebral hemorrhage. Stroke 2014; 45: 3151-52.

40. Rosand J, Hylek E, O’Donnell H, Greenberg S. Warfarin-associated hemorrhage and cerebral amyloid angiopathy a genetic and pathologic study. Neurology 2000; 55: 947-51.

41. Lee S, Ryu W, Roh J. Cerebral microbleeds are a risk factor for warfarin-related intracerebral hemorrhage. Neurology 2009; 72: 171-6.

42. Wilson D, Ambler G, Shakeshaft C, Brown M, Charidimou A, Salman R, et al. Cerebral microbleeds and intracranial haemorrhage risk in patients anticoagulated for atrial fibrillation after acute ischemic stroke or transient ischemic attack (CROMIS-2): a multicenter observational study. Lancet Neurol 2018; 17: 539-47.

43. Eckman MH, Wong LK, Soo YO, Lam W, Yang SR, Greenberg SM, et al. Patient-specific decision-making for warfarine therapy in nonvalvular atrial fibrillation how will screening with genetics and imaging help? Stroke 2008; 39: 3308-15.

44. Tsivgoulis G, Zand R, Katsanos A, Turc G, Nolte C, Jung $\mathrm{S}$, et al. Risk of symptomatic intracerebral hemorrhage after intravenous thrombolysis in patients with acute ischemic stroke and high cerebral microbleed burden a meta-analysis. JAMA Neurol 2016; 73 (6): 675-83.

45. Dannenberg S, Scheitz J, Rozanski M, Erdur H, Brunecker $\mathrm{P}$, Werring $\mathrm{D}$, et al. Number of cerebral microbleeds and the risk of intracerebral hemorrhage after intravenous thrombolysis. Stroke 2014; 45: 2900-5.

46. McCarron M, Nicoll J. Cerebral amyloid angiopathy and thrombolysis-related intracerebral haemorrhage. Lancet Neurol 2004; 3: 484-92.

47. Powers W, Rabistein A, Ackerson T, Adeoye O, Bambakidis N, Becker K, et al. 2018 Guidelines for the early management of patients with acute ischemic stroke. Stroke 2018; 49: e46-e99.

48. Viswanathan A, Rakich S, Engel C, Snider R, Rosand J, Greenberg S, et al. Antiplatelet use after intracerebral hemorrhage Neurology 2006;66: 206-9.

49. Biffi A, Halpin A, Towfighi A, Gilson A, Busl K, Rost N, et al. Aspirin and recurrent intracerebral hemorrhage in cerebral amyloid angiopathy. Neurology 2010; 75: 693-8.
50. O’Donnell H, Rosand J, Knudsen K, Furie K, Segal A. Chiu R, et al. Apolipoprotein E genotype and the risk of recurrent lobar intracerebral hemorrhage. N Eng J Med 2000; 342: 240-5.

51. Charidimou A, Peeters A, Jäger R, Fox Z, Vandermeeren $\mathrm{Y}$, Laloux $\mathrm{P}$, et al. Cortical superficial siderosis and intracerebral hemorrhage risk in cerebral amyloid angiopathy. Neurology 2013; 81: 1666-73.

52. Greenberg S, Eng J, Ning M, Smith E, Rosand J. Hemorrhage burden predicts recurrent intracerebral hemorrhage after lobar hemorrhage. Stroke 2004; 35: 1415-20.

53. Hemphil C, Greenberg S, Anderson C, Recker K, Benbok B, Cushmann M, et al. Guidelines for the management of spontaneous intracerebral hemorrhage. Stroke 2015; 46: 2032-80.

54. Eckman M, Rosand J, Knudsen K, Singer D, Greenberg S. Can patients be anticoagulated after intracerebral hemorrhage? A decision analysis. Stroke 2003; 34: 1710-6.

55. Biffi A, Kuramatsu J, Leasure A, Kamel H, Kourkoulis C, Schwab K, et al. Oral Anticoagulation and Functional Outcome after Intracerebral Hemorrhage. Ann Neurol 2017; 82: 755-65.

56. Biffi A, Anderson C, Battey T, Ayres A, Greenberg S, Viswanathan A, et al. Association between blood pressure control and risk of recurrent intracerebral hemorrhage. JAMA 2015; 314 (9): 904-12.

57. Izumihara A, Ishihara $\mathrm{T}$, Iwamoto $\mathrm{N}$, Yamashita $\mathrm{K}$, Ito $\mathrm{H}$. Postoperative outcome of 37 patients with lobar intracerebral hemorrhage related to cerebral amyloid angiopathy. Stroke 1999; 30: 29-33.

58. Charidimou A, Boulouis G, Haley K, Auriel E, van Etten E, ForiadisP, et al. White matter hyperintensity patterns in cerebral amyloid angiopathy and hypertensive arteriopathy. Neurology 2016; 86: 1-7.

59. Gregorie S, Charidimou A, Gadapa N, Dolan E, Antoun $\mathrm{N}$, Peeters $\mathrm{A}$, et al. Acute ischaemic brain lesions in intracerebral haemorrhage: multicenter cross-sectional magnetic resonance imaging study. Brain 2011; 134: 2376-86.

60. Gurol M, Becker J, Fotiadis P, Riley G, Schwab K, Johnson K, et al. Florbetapir-PET to diagnose cerebral amyloid angiopathy: A prospective study. Neurology 2016; 87: 2043-9.

61. Renard D, Castelnovo G, Wacongne A, Le Floch A, Thouvenot E, Mas J, et al. Interest of CSF biomarker analysis in possible cerebral amyloid angiopathy cases defined by the modified Boston criteria. J Neurol 2012; 259: 2429-33. 